\title{
Economias de escala na produção de leite: uma análise dos Estados de Rondônia, Tocantins e Rio de Janeiro
}

\author{
Alexandre Lopes Gomes* \\ Joaquim Bento de Souza Ferreira Filho**
}

Resumo: Neste trabalho analisou-se o problema da manutenção, no longo prazo, dos produtores de leite na atividade. Foram analisados estabelecimentos nos estados de Rondônia, Tocantins e Rio de Janeiro. O objetivo do trabalho foi verificar a existência de economias de escala entre os produtores de leite. A função que apresentou melhor aderência aos dados foi a de custo translog. Os fatores de produção considerados foram: capital, terra, trabalho e custeio. A análise econômica mostra a dificuldade de sobrevivência dos estabelecimentos no longo prazo. Isto ocorre porque a relação capital imobilizado/produção é muito alta. Os resultados da regressão revelam que a grande maioria dos produtores da amostra está na faixa de economias de escala, sendo que apenas 3,4\% destes estão na faixa de deseconomias de escala. O ponto de custo médio mínimo foi obtido com média diária de 487 litros por dia. Outro grupo composto por $10 \%$ da amostra está mais próximo do ponto de custo médio mínimo e apresenta produção diária entre 183 e 487 litros por dia. E finalmente, o último grupo apresentando uma produção inferior a 180 litros/dia. Este grupo pode reduzir de forma significativa os custos aumentando a produção e, usufruindo assim das economias de escala.

\footnotetext{
* Doutor em Economia Aplicada, Escola Superior de Agricultura "Luiz de Queiroz" - ESALQ/USP e Professor Adjunto da Universidade Federal Fluminense. algomes@metal.eeimvr.uff.br

**Livre Docente pela Universidade de São Paulo. jbsferre@esalq.usp.br
} 
Economias de escala na produção de leite: uma análise dos Estados de Rondônia, Tocantins e Rio de Janeiro

Palavras-chave: Produção de leite; Economias de escala; Função custo

Classificação JEL: D-24

\begin{abstract}
This study aimed to analize the business maintenance issue faced by milk producers, in the long term. It was analized farms in the states of Rondônia, Tocantins and Rio de Janeiro. The main goal of this study was to verify the existence of economies of scale among milk producers. The function that presented better adequacy to the data was that of cost translog. The production factors taken into consideration were: capital, land, work and direct expenses. The economic analysis shows the difficulties to survive faced by farmers in the long term. This happens because the relation immobilized capital/production is very high. The regression results reveal that the vast majority of producers in this case study operate in economies of scale, being that only $3.4 \%$ of them do not. The point of minimum average cost was obtained at about 178 thousand liters per year, that is, an average of 487 liters per day. Another group formed by $10 \%$ of the case study is closer to the point of minimum average cost and presents a daily production between 183 and 487 liters. And finally, the last group which presents itself in a less favorable situation with a production lower than 180 liters per day. It is highlighted that these producers can significantly reduce their costs if they increase their production, once they are found in the most accentuated part of the average cost curve in the long term and can greatly develop in the activity by using the economies of scale.
\end{abstract}

Key words: Milk production; Economies of scale; Cost function

JEL Classification: D-24

\title{
1. Introdução
}

No Brasil, o complexo agroindustrial do leite tem importância significativa, está presente em todo território nacional e, na maioria dos estados, gera renda e uma quantidade expressiva de postos de trabalho. Segundo o Censo Agropecuário de 1996 (INSTITUTO BRASILEIRO DE GEOGRAFIA E 
ESTATISTICA - IBGE, 1996), do total de 4,83 milhões de estabelecimentos agropecuários do Brasil, 1,81 milhões dedicavam-se, ao menos parcialmente, à pecuária de leite, o que representava $37 \%$ do total.

O Brasil é sexto maior produtor de leite do mundo com 23,5 bilhões de litros produzidos no ano de 2004 (IBGE, 2005). Segundo Gomes (2001), a década de 1990 foi um "divisor de águas” para a cadeia agroindustrial do leite no Brasil, em função de três fatores que foram fundamentais para as transformações observadas no setor: 1) Liberação do preço do leite, em setembro de 1991; 2) Estabilidade da economia, com destaque para a queda da inflação com a implantação do Plano Real, em julho de 1994; e 3 ) Maior abertura comercial. Estes fatos foram responsáveis para que a cadeia do leite tenha sido uma das que mais se transformou nos últimos anos. Entre estas transformações podemse destacar o crescimento expressivo da produção nacional, principalmente na região Centro-Oeste, assim como um aumento significativo das importações e queda de preços com conseqüente redução da renda líquida dos produtores.

\subsection{Transformações da pecuária de leite no Brasil}

Em 1991 houve o fim do tabelamento de preços após 46 anos de regulamentação do Estado. Segundo Martins (2002), o argumento para a intervenção do Estado no preço do leite sempre foi a proteção dos consumidores, e acentuou-se na década de 1980, quando o controle do custo de vida recebeu maior atenção em função das altas taxas de inflação. No entanto, os resultados do longo período do tabelamento não foram satisfatórios. Em nível de produção, o que se observou foram taxas reduzidas de crescimento da produção acompanhada por baixos índices zootécnicos, pouca especialização do rebanho e restrita adoção de práticas higiênico-sanitárias.

O Plano Real, em 1994, fez com que as importações de leite aumentassem de forma significativa, especialmente de leite em pó. Este fenômeno teria ocorrido para cobrir os déficits gerados pela elevação da renda per capita, impedindo que os preços internos se elevassem. Vale lembrar que, em equivalentes litros de leite, as importações corresponderam, em 1995, a cerca de $20 \%$ da produção nacional e $13 \%$ em 1996, Gomes (2002). 
A prática de dumping no leite exportado para o Brasil, derrubava o preço doméstico, além de contribuir para ampliar sua instabilidade, na medida em que ocorriam maciças importações também no período de safra da produção nacional. A queda de preço do leite e o aumento de sua instabilidade inviabilizaram vários projetos de produção de leite, tendo como conseqüência natural, a expulsão de produtores e de seus empregados, o que significou a saída prematura do homem do campo. Este processo vem ocorrendo até hoje, mesmo que em menor intensidade.

Outro fator que merece destaque neste processo de transformação no setor diz respeito à abertura da economia brasileira e à criação do Mercosul no início da década de 1990. Estes fatos proporcionaram uma série de transformações no setor, já que o segmento lácteo se viu exposto à concorrência de empresas de grande porte e dos preços praticados no mercado internacional. Assim, a abertura comercial fez com que os produtores nacionais tivessem que concorrer com produtores de outros países, situação desconhecida pelo produtor nacional uma vez que o mercado era controlado pelo governo. A situação de baixa produtividade do rebanho, a reduzida produção por propriedade e a qualidade inferior do leite entregue aos laticínios foram apontados por Faria (1995) e Rodrigues (1999) como restrições a serem vencidas, visando aumentar a competitividade do setor frente aos produtos importados.

Vale lembrar que esta competição internacional muitas vezes não ocorreu de forma justa, pois na década de 1990 observou-se importação de leite subsidiado no país de origem, penalizando o produtor, uma vez que o leite entrava no Brasil a um custo inferior ao custo de produção local.

As transformações estruturais que ocorreram no setor, alavancadas pela magnitude das importações na década de 1990 e pela prática de subsídios no mercado lácteo internacional, favoreceram a queda da renda líquida do produtor de leite no Brasil. No entanto, de acordo com Gomes (2001) este fenômeno pode ser explicado também pela deterioração dos termos de troca (preço recebido/preço pago). Esta deterioração pode ser justificada pelos seguintes fatores: 1) redução do preço recebido para dado preço pago; 2) redução do preço recebido mais que proporcional à queda do preço pago; e 3) aumento do preço pago para dado preço recebido.

Gomes (2001) mostra ainda que, além dos termos de troca, dois ou- 
tros elementos podem influenciar a renda do produtor: produtividade e volume de produção/empresa rural. A produtividade, medida em litros de leite/vaca ordenhada, cresceu 5,40\% na década de 1990. É verdade que a redução do número de vacas a uma taxa de 2,0\% ao ano contribuiu para o crescimento da produtividade. Mas não se pode negar que a produtividade aumentou nesse mesmo período, principalmente, pela utilização de sistemas mais intensivos de produção, o que promoveu uma maior produção por animal.

Quanto ao volume médio de produção por empresa rural, este de fato aumentou, especialmente após 1998, quando a adoção do resfriamento do leite na fazenda e o transporte a granel ficaram mais comuns. Aqui também prevalece a mesma lógica do aumento da produtividade, onde o produtor pouco se beneficiou dos aumentos de volume de produção, porque eles apenas compensaram parte das perdas decorrentes da queda dos termos de troca.

Em resumo, os termos de troca caíram significativamente, a produtividade (de modo mais expressivo em termos percentuais que em valores absolutos) e o volume de produção/empresa aumentaram. Contudo, os ganhos de produtividade e o aumento da produção não foram suficientes para neutralizar a queda dos termos de troca. A resultante deste processo foi uma diminuição da renda líquida e o empobrecimento do produtor.

\subsection{Considerações sobre eficiência e economias de escala}

A renda líquida de longo prazo é um indicador de sobrevivência do estabelecimento. Segundo Alves et al. (2001), ela mede a remuneração do empreendedor pelo risco que corre de administrar o estabelecimento. É o resíduo que sobra depois de se remunerar todos os fatores de produção. O empreendedor compara essa renda com outras alternativas para verificar se compensa manter o estabelecimento em funcionamento. Em suma, pode-se dizer que a renda líquida mede a estabilidade do estabelecimento. A queda da renda líquida do produtor de leite nos últimos anos fez com que muitos produtores deixassem a atividade, o que levou a uma redução expressiva no seu número.

Gomes (2001) aponta que a redução do preço das importações de 
Economias de escala na produção de leite: uma análise dos Estados de Rondônia, Tocantins e Rio de Janeiro

leite favoreceu queda dos preços domésticos e contribuiu para a exclusão de produtores. A rapidez com que esta exclusão ocorreu gerou profundas conseqüências sociais, na medida em que o produtor, ao abandonar a atividade, reduz a demanda por mão-de-obra no campo.

No entanto, Souza (2002) mostra que além do preço recebido, a eficiência alocativa e o nível de tecnologia utilizado no sistema de produção, também contribuem para aumentar a renda líquida do produtor. A escolha errada da cesta de insumos ou da tecnologia torna o estabelecimento pouco competitivo, reduz as suas receitas e pode comprometer a manutenção do produtor na atividade. A competição existente no mercado exige que o produtor esteja sempre buscando a maior eficiência possível dos fatores de produção utilizados na atividade.

Pereira Filho (2000), afirma que embora a competitividade de uma firma dependa de fatores microeconômicos e macroeconômicos, a análise de eficiência baseada em fatores microeconômicos é de grande relevância para a explicação da competitividade. Pode-se ainda pressupor que a competitividade caminha lado a lado com a obtenção de renda líquida positiva, fato que gera uma perspectiva de crescimento e longevidade para estabelecimentos competitivos.

Alves et al. (2001) analisaram 963 estabelecimentos em todo território nacional, todos com área menor que 100 hectares e tentaram explicar a causa de $74 \%$ dos mesmos terem apresentado renda líquida negativa. A análise foi feita com base nos insumos terra, capital e trabalho. Uma das conclusões observadas no trabalho é que a má alocação de recursos contribuiu de forma significativa para determinar a existência de renda líquida negativa na maior parte da amostra.

Alves (2004), analisando a existência de economias de escala em cerca de 1,8 milhões de estabelecimentos agrícolas, verificou que aqueles com menores níveis de produção e renda apresentavam retornos crescentes à escala. Segundo o autor, estes produtores enfrentam imperfeições do mercado para otimizarem o nível de produção e por isso, muitas vezes, se mantêm à margem do processo de modernização da agricultura.

Gomes et al. (2005), analisando produtores de leite dos estados de Rondônia, Tocantins e Rio de Janeiro mostraram que existe uma grande dificuldade em identificar os fatores que tornam os produtores mais ou menos eficientes na produção. No entanto, utilizando o modelo de 
fronteiras eficientes e um conjunto de insumos pôde-se notar pelos autores que os produtores ineficientes podem diminuir a quantidade de insumos utilizados com o objetivo de reduzir a ineficiência.

Alves (2001) confirmou o excesso no uso de insumos, especialmente de mão-de-obra, na produção de leite ao analisar produtores no Estado de Minas Gerais. De acordo com o referido autor, custos elevados podem ser evitados reduzindo-se o número de trabalhadores. No entanto, observa-se que a estratégia utilizada para reduzir os custos com mão-de-obra se constitui na prática de baixos salários. Por outro lado, a otimização da mão-de-obra poderia permitir a redução dos custos e o pagamento de salários mais justos aos empregados. O autor mostra ainda que os produtores não minimizam o custo médio em função de não disporem de recursos para realizar a composição ótima dos fatores produtivos.

Ferreira (2002) ao analisar um grupo de produtores no Estado de Minas Gerais, na tentativa de determinar fatores de produção que poderiam estar afetando o nível de eficiência sinalizou que os produtores ineficientes deveriam minimizar o uso de concentrados e mão-de-obra. $\mathrm{O}$ autor verificou também que os produtores menos eficientes apresentavam investimentos em máquinas e terra além do necessário para o nível de produção observado e ainda que a maior escala de produção é sempre desejada pelos produtores, no entanto não garante a eficiência técnica.

Roberts et al. (2004) analisando produtores no Estado de Rondônia verificou a elevada participação da mão-de-obra familiar em todos os sistemas de produção, o que reflete a importância da atividade na ocupação do trabalho familiar. Os autores mostram que os produtores com produção diária de até 50 litros estavam trabalhando na faixa de economias de escala, sinalizando a possibilidade de crescimento de pequenas propriedades. Entretanto, constatou-se também que existem relações negativas entre a eficiência de escala e as variáveis número de vacas, mão-de-obra e gastos operacionais totais. Em síntese, embora a eficiência técnica dos pequenos produtores não seja significativamente diferente dos demais, há o problema da ineficiência de escala.

Dentro deste contexto de escala de produção, eficiência no uso dos insumos e o diagnóstico de fatores que determinam a sustentabilidade dos produtores de leite no longo prazo, o foco de análise deste trabalho são os estados de Rondônia, Tocantins e Rio de Janeiro. Sabe-se que 
a pecuária de leite, nas pequenas propriedades, e a de corte, nas grandes, expandiu muito nos últimos anos. Entre as regiões do país, Gomes (2002) destaca que os maiores crescimentos no período de 1994 a 2003 aconteceram no Norte $(10,43 \%$ ao ano) e no Centro-Oeste $(5,23 \%$ ao ano). Em relação à participação da produção na região Norte, o estado que mais aumentou sua participação foi Rondônia, passando de $25,83 \%$ para $40,90 \%$ no mesmo período.

De 1990 a 2000, enquanto a produção de leite do Brasil cresceu à taxa de $3,19 \%$ ao ano, em Rondônia a produção cresceu 6,01\%, e em Tocantins 4,16\% ao ano. A predominância do clima tropical e úmido tem grande influência na definição dos sistemas de produção de leite adotados nestes estados. O desempenho da produção de leite na região Norte nos últimos anos é explicado pela abundância de chuvas, pouca exigência de mão-de-obra e mercado garantido.

Dada esta importância social e econômica da atividade leiteira nestes três estados, a identificação da existência ou não de economias de escala na produção de leite, bem como a análise dos fatores que a determinam, são de fundamental importância.

Portanto, o objetivo do trabalho é analisar a existência de economias de escala entre os produtores de leite dos estados de Rondônia, Tocantins e Rio de Janeiro.

Estes indicadores permitem analisar as possibilidades de sobrevivência dos estabelecimentos, dada a restrição de recursos que caracteriza a pequena propriedade e sua dependência da atividade leiteira como fonte de renda.

\section{Metodologia}

\subsection{Definição da função custo}

O referencial teórico utilizado para obter as elasticidades de produção é a função de produção ou função custo. Ambas podem ser usadas neste caso devido à dualidade existente entre estas duas funções.

Dentro deste contexto, Binswanger (1974) mostra que a função custo apresenta certas vantagens em relação à função de produção. Dentre elas está a homogeneidade nos preços dos fatores, independente 
das propriedades de homogeneidade da função de produção; a variável preço assume o papel de variável explicativa, ao contrário da função de produção quando as quantidades dos fatores é que são as variáveis independentes; na função custo obtém-se diretamente as elasticidades de substituição entre os fatores (na função de produção é necessário fazer inversão de matrizes dos coeficientes da função); e ainda, na função custo reduzem-se os problemas de multicolinearidade que podem ocorrer na função de produção.

Chambers (1994) define a função custo pela seguinte expressão:

$$
c(w, y)=\min _{x \geq 0}\left\{w^{*} x: x \in V(y)\right\},
$$

onde $\mathrm{w}$ representa os preços dos insumos e $\mathrm{w}_{\mathrm{i}}{ }^{*} \mathrm{X}_{\mathrm{i}}$ representa as despesas com cada insumo i. O somatório destas despesas $\left(\sum_{i} w_{i} x_{i}\right)$ representa o custo total que deverá ser minimizado. Portanto, a função custo é o custo mínimo de se produzir determinado nível de produto, durante um período de tempo, expresso em função dos preços dos insumos e da quantidade a ser produzida. A representação citada assume que os preços dos insumos são exógenos.

\subsection{A Função Custo Transcendental Logarítmica}

Pela teoria da dualidade, a partir da função de produção agregada pode-se obter a função custo correspondente. O método utilizado para obter os resultados foi proposto por Binswanger (1974) e utilizado ainda por outros autores, que mostra no caso da firma que utiliza $n$ insumos, a função custo definida em (1). A função custo minimizada pode ser representada por:

$$
C^{*}=c\left(y, w_{1}, w_{2}, \ldots, w_{n}\right)
$$

em que, C* é o custo mínimo de se produzir o nível de produção $\mathrm{y}, \mathrm{e}_{\mathrm{i}}$ representa os preços dos insumos. Assim, aplicando logaritmos naturais à expressão (21) e expandindo-se por meio de uma série de Taylor $^{1}$ de segunda ordem, tem-se a função de custo translog:

${ }^{1}$ Para expansão por meio de uma série de Taylor ver Chiang (2004) 


$$
\ln C^{*}(w, y)=\beta_{0}+\sum_{i=1}^{n} \beta_{i} \ln w_{i}+\beta_{y} \ln y+\frac{1}{2} \sum_{i=1}^{n} \sum_{j=1}^{n} \beta_{i j} \ln w_{i} \ln w_{j}+\sum_{i=1}^{n} \beta_{i j} \ln w_{i} \ln y_{i}+\beta_{y y}(\ln y)^{2}
$$

em que os $\beta$ 's são os parâmetros estruturais da função de custo e os índices i e j representam os fatores de produção. De acordo com o Teorema de Young, pode-se considerar as seguintes condições de simetria $\beta_{i j}=\beta_{j i}$ para $i \neq j$ em relação aos parâmetros estimados na função translogarítmica. Ou seja, a taxa marginal de substituição técnica do insumo i pelo insumo j é igual à taxa de j pelo insumo i. A homogeneidade linear nos preços dos fatores é garantida pelas seguintes relações entre os parâmetros:

$$
\sum_{i=1}^{n} \beta_{i}=1 \text { e } \sum_{i=1}^{n} \beta_{i y}=\sum_{i=1}^{n} \beta_{i j}=\sum_{j=1}^{n} \beta_{i j}=\sum_{i=1}^{n} \sum_{j=1}^{n} \beta_{i j}=0
$$

As condições de monotonicidade e concavidade são conferidas localmente. Para garantir a monotonicidade, as parcelas de custo devem ser não-negativas, e a concavidade será atendida se o determinante da matriz hessiana for negativo. Aplicando a derivada na função custo em relação aos preços dos fatores tem-se, pelo Lemma de Shephard, a demanda derivada de cada fator.

$$
\frac{\partial C^{*}}{\partial w_{i}}=x_{i}
$$

Assim, os parâmetros da função custo podem ser obtidos por meio da estimação de um sistema de $n$ equações compostas pelas parcelas de custo.

$$
\frac{\partial \ln C^{*}}{\partial \ln w_{i}}=S_{i}=\beta_{i}+\sum_{j=1}^{n} \beta_{i j} \ln w_{j}+\beta_{i y} \ln y
$$

A expressão 6 representa a participação do insumo i no custo total, e como já citado, deve ser não-negativa para garantir a monotonicidade da função custo.

$$
\frac{\partial \ln C^{*}}{\partial \ln w_{i}}=S_{i}>0
$$


Segundo Christensen e Greene (1976), apesar da função translog não obrigar a estrutura de produção a ser homotética e nem impor restrições às elasticidades de substituição, estas condições podem ser testadas estatisticamente:

- Uma função custo corresponde a uma função de produção homotética, se e somente se, puder ser separável em produção e preços dos fatores. Assim, uma função translog de produção estará associada a uma função de produção homotética se:

$$
\beta_{i y}=0(\mathrm{i}=1,2, \ldots, \mathrm{n})
$$

- Uma estrutura de produção homotética é também homogênea, se e somente se, a elasticidade do custo com respeito à produção é constante. Desta forma, a função de custo estará associada a uma função de produção homogênea se:

$$
\beta_{i y}=0(\mathrm{i}=1,2, \ldots, \mathrm{n}) \text { e } \beta_{y y}=0
$$

A elasticidade de substituição pode ser unitária entre os fatores de produção se os termos de segunda ordem nos preços forem retirados do modelo. A restrição para elasticidade unitária na função custo translog é dada pela seguinte forma:

$$
\beta_{i j}=0(\mathrm{i}=1,2, \ldots, \mathrm{n})
$$

Deve-se observar que quando $\beta_{i y}=0, \beta_{y y}=0, \beta_{i j}=0$ e $\sum \beta_{i}=1$, a função de custo translog se reduz a uma função de custo do tipo CobbDouglas, como um caso particular.

\subsection{Metodologia de estimação da função custo translogarítmica}

Os parâmetros da função translog podem ser estimados pelo método usual de mínimos quadrados ordinários. No entanto, Christensen e Greene (1976) argumentam que esta técnica pode negligenciar as informações adicionais contidas nas equações de parcelas de custos, que também são facilmente estimáveis. 
Economias de escala na produção de leite: uma análise dos Estados de Rondônia, Tocantins e Rio de Janeiro

Assim, o procedimento de estimação utilizado será o descrito por Greene (2000), onde se calcula conjuntamente a função custo total translog e as equações de parcelas de custo como um sistema de regressão multivariada. A estimativa conjunta das funções de custo total translog e de parcelas de custo, resultam em parâmetros mais eficientes do que aqueles que seriam obtidos aplicando-se M.Q.O. para a função custo de forma isolada.

Esta metodologia foi utilizada em trabalhos como de Christensen e Greene (1976), Ray (1982), Albuquerque (1987) e Garcia (2004). Assume-se que os erros têm distribuição normal e são aditivos para cada uma das equações de parcela de custos e para a função de custo.

São impostas condições de simetria e homogeneidade por meio da normalização da função custo total translog. Deve-se deixar de fora da estimação uma equação de parcela de custo para evitar o problema de singularidade da matriz de variância e covariância dos erros. Estima-se assim, o restante do sistema e obtém-se o restante dos parâmetros por diferença. Em princípio é irrelevante qual fator será excluído. Greene (2000) mostra que os parâmetros obtidos não variam em relação à equação a ser excluída deixando-se interagir as estimativas de Mínimos Quadrados Generalizados, ou estimando-se o modelo por máxima verossimilhança dos parâmetros para assegurar a invariância com respeito à parcela de custo a ser deixada de fora do modelo.

As variáveis utilizadas foram: capital $(\mathrm{K})$, terra $(\mathrm{T})$, mão-de-obra (M) e dispêndio (D). Este último item envolve dispêndios diretos com alimentação dos animais, medicamentos e energia elétrica utilizada no estabelecimento. O sistema de equações, sem restrições, composto pelas funções de custo translog e de parcelas de custo é representado por:

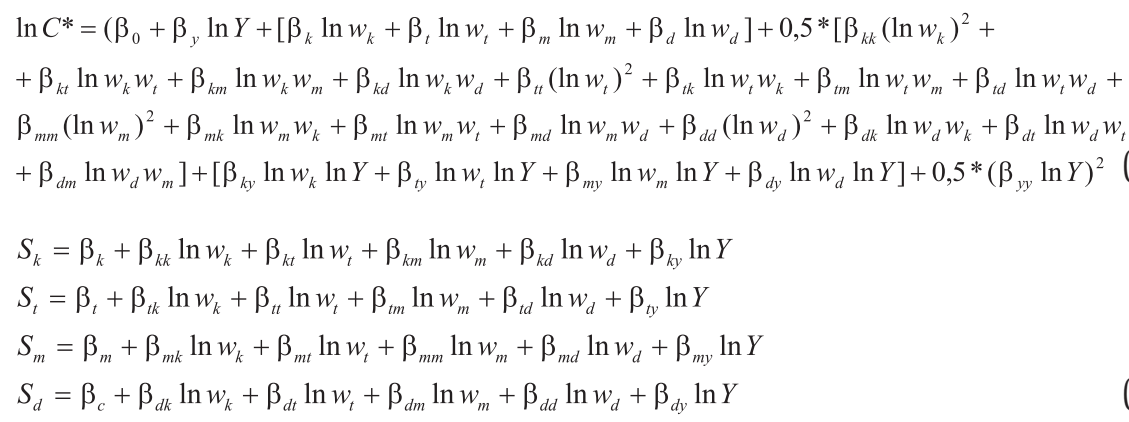


Em que $S_{k} S_{t} S_{m} S_{d}$ referem-se às parcelas de gastos com capital, terra, mão-de-obra e custeio, respectivamente. Y é a produção de leite, em litros, e $w_{k}, w_{t}, w_{m}$ e $w_{d}$ representam os preços dos insumos (em $\mathrm{R} \$$ ).

\subsection{Descrição das variáveis}

Nesta seção são descritas as variáveis utilizadas na estimação da função de custo translog.

$>$ Capital: considerou-se como capital na atividade leiteira das propriedades analisadas as benfeitorias, máquinas e equipamentos, animais de produção e serviços, além de forrageiras não naturais. O preço do capital utilizado na função custo foi obtido pela relação entre o valor de fluxo, capaz de medir o valor dos serviços prestados pelo capital em um ano (depreciação + juros), e o valor de estoque de capital.

- Benfeitorias: em relação a este indicador de fluxo considerouse o valor de $6 \%$ sobre o capital médio, que foi obtido pelo valor do capital inicial somado ao capital final dividido por dois. O valor de estoque de capital na propriedade pode ser estimado pelo valor novo do equipamento multiplicado pela sua percentagem de uso na atividade. Dividindo-se o valor de fluxo pelo valor de estoque tem-se o preço das benfeitorias que compõem o preço do capital. Foram considerados como benfeitorias no levantamento dos dados: curral, sala de ordenha, estábulo, tronco, silo, bezerreiro, sala de máquinas, estradas perimetrais e internas, outros.

- Máquinas e equipamentos: a estimação deste item foi feita de forma semelhante ao processo utilizado no item benfeitoria. Gerou-se um valor de fluxo a partir dos juros mais a depreciação e um valor de estoque. Por esta relação obteve-se o preço de máquinas e equipamentos que compôs o preço do capital. Foram considerados como máquinas e equipamentos: picadeira, pulverizador, resfriador, botijão de sêmen, carroça, ordenhadeira mecânica, ensiladeira, trator, arado, grade, balança, equipamento para irrigação, utensílios, motocicleta e outros.

- Animais: em relação aos animais aplicou-se uma taxa de $6 \%$ 
sobre o valor de estoque dos animais a fim de se obter um fluxo anual de despesas correspondentes aos mesmos.

- Forrageiras não naturais: neste item foi utilizado o valor gasto na manutenção das forrageiras não naturais e incorporado ao item capital como sendo o preço do serviço no ano corrente.

Depreciação: as estimativas de depreciação foram efetuadas pelo método linear que considera a depreciação proporcional do capital ao longo de sua vida útil. Portanto, obtém-se esta taxa por meio da diferença entre o valor novo e o residual dividido pelo número de anos. Considerou-se o valor residual das benfeitorias, máquinas e equipamentos e animais igual a zero ao final da vida útil dos bens. Este procedimento foi sugerido por Buarque (1991).

$>$ Terra: adotou-se o procedimento seguido por Alves; Souza e Brandão (2001), de imputar ao custo de oportunidade da terra $4 \%$ sobre seu valor. Assim pode-se gerar um valor de fluxo anual de gastos referentes a este fator.

Mão-de-obra: considera-se o somatório das despesas com mãode-obra contratada e mão-de-obra familiar dividido pelo número de dias trabalhados. Assim se obteve o preço da mão-de-obra.

Dispêndio: A variável dispêndio representa as despesas diretas com alimentação dos animais, despesas com medicamentos e energia elétrica.

\subsection{Fonte de dados}

Os dados utilizados neste trabalho foram coletados na Implantação do "Programa Cadeias Produtivas Agroindustriais" do Sebrae Nacional entre os anos de 2002 a 2004, pela equipe do Professor Sebastião Teixeira Gomes, da Universidade Federal de Viçosa. Este programa tem como objetivo o estudo da Cadeia Produtiva do Leite nos estados de Rondônia, Tocantins e Rio de Janeiro, e foi desenvolvido em parceria com o Programa Estadual de Melhoria da Produção e Produtividade da Pecuária Leiteira (PROLEITE), da Câmara Setorial e demais instituições envolvidas no setor. 


\subsection{Seleção dos produtores de leite}

\subsubsection{No Estado de Rondônia}

Para elaboração do diagnóstico da produção de leite em Rondônia foram coletados dados, em pesquisa de campo, de uma amostra de 457 produtores de leite. A amostra cobriu todos os municípios que produziam mais de $2 \%$ da produção de leite neste estado, segundo dados do IBGE (2000). A amostra foi dividida de acordo com a participação de cada município na produção do estado. Por exemplo, 20,43\% dos entrevistados estavam localizados no município de Ouro Preto do Oeste.

Para o cálculo da amostra foi solicitado a todos os laticínios de Rondônia uma lista de seus fornecedores, com as respectivas produções. No entanto, alguns laticínios não apresentaram suas listas. Por essa razão, a estratificação da amostra foi feita a partir de uma população de 2.370 produtores que forneciam leite aos laticínios, os quais apresentaram as listas de seus fornecedores. Na opinião de especialistas na produção de leite em Rondônia, os laticínios que apresentaram as listas de fornecedores representam, com boa proximidade, a realidade estadual.

A amostra foi também estratificada de acordo com a distribuição do número de produtores de leite da população. Por exemplo, $64 \%$ da amostra foi composta por produtores que produziam até 50 litros de leite/dia, e apenas $5 \%$ produziam mais que 200 litros/dia. No dimensionamento da amostra considerou-se a produção comercializada pelo mercado de leite inspecionado, em Rondônia, em 2001. A partir dos dados da Pesquisa Trimestral do Leite, do IBGE, o mercado formal de leite em Rondônia foi estimado em 776.388 litros/dia e a média diária por produtor foi de 68 litros. Portanto, verifica-se que o número total de produtores de leite do mercado formal de Rondônia era de 11.417 (776.388 litros/dia $\div 68$ litros/produtor/dia).

Do universo de 11.417 produtores foram entrevistados 457 que correspondiam a $4 \%$ da população. Tal percentual está dentro dos limites permitidos em pesquisas dessa natureza, visto que, em um mesmo estrato, os sistemas de produção não apresentam grandes variações, pois estas ocorrem entre estratos de produção.

A amostra de 457 produtores foi dividida, inicialmente, entre os 
municípios, de acordo com a participação (\%) destes na produção do estado. A seguir, a amostra de um município foi dividida em estratos de produção, de acordo com a distribuição da população. Finalmente, a amostra foi dividida em dois grupos; o primeiro constituído de 114 produtores $(25 \%)$, aos quais foram aplicados questionários quantitativos que objetivavam a análise financeira dos sistemas de produção; e o segundo de 343 produtores (75\%), aos quais foram aplicados questionários qualitativos que visavam caracterizar os perfis dos sistemas de produção de leite em Rondônia.

A aplicação dos questionários aos produtores foi feita no período de 11 de junho a 5 de julho de 2002. Os dados levantados referem-se ao ano 2001/2002, especificamente, de junho de 2001 a maio de 2002. Foram utilizados preços médios de insumos, serviços e produtos referentes a esse período.

Os questionários foram aplicados pelos técnicos da EMATER-RO, com supervisão de dois veterinários contratados. Os produtores foram entrevistados em suas propriedades, de modo que os entrevistadores pudessem avaliar as respostas dadas.

\subsubsection{No Estado de Tocantins}

Os dados utilizados no trabalho, em nível de empresa rural produtora de leite, da região central do Estado de Tocantins, foram obtidos a partir da aplicação de questionários em uma amostra de 292 produtores de leite, que corresponde a $5 \%$ do universo de produtores desta região.

A amostra foi distribuída entre os municípios da região central do Estado de Tocantins, na mesma proporção da produção do universo de produtores.

A cidade de Paraíso do Tocantins foi a que apresentou maior produção entre os municípios amostrados, com 66 produtores. A amostra foi estratificada pela produtividade (litros/vaca em lactação/dia). Nota-se que $40 \%$ dos estabelecimentos apresentaram média de produtividade abaixo de 3 litros por vaca. Este grupo de produtores apresentou uma média de produção de 47,62 litros/dia, o que representa apenas 24,44\% do total produzido pelos entrevistados.

Os dados levantados junto aos produtores referem-se aos anos 
de 2003/2004, especificamente de agosto de 2003 a julho de 2004 . Foram utilizados preços médios dos produtores, insumos e serviços deste período.

\subsubsection{No Estado do Rio de Janeiro}

No dimensionamento da amostra estratificada dos produtores a serem entrevistados foram utilizados três grupos: a) número total de produtores de leite no Estado do Rio de Janeiro que fazem parte do mercado inspecionado; b) distribuição da produção de leite, no Estado do Rio de Janeiro, entre os municípios e c) distribuição da produção de leite, no Estado do Rio de Janeiro, nos estratos de produção. Ao analisar o número de produtores de leite do Estado do Rio de Janeiro e a sua distribuição nas principais regiões do estado observou-se uma maior concentração de produtores nas regiões Centro-Sul e Noroeste, em torno de $50 \%$ do total de produtores do estado. A região com menor número de produtores é a litorânea.

Em relação à distribuição da produção de leite nas principais regiões do Estado do Rio de Janeiro, nota-se que a região Centro-Sul apresenta a maior participação na produção com $35,2 \%$ do total produzido no estado. Em segundo lugar aparece a região Noroeste com 24,0\%. As regiões Norte, Serrana e Litorânea estão no mesmo patamar e são as que menos produzem leite, apresentando contribuições que variam de 13 a $14 \%$ da produção total do estado.

Quanto à distribuição dos produtores em estratos de produção, observa-se que $41,93 \%$ dos produtores produzem menos de 30 litros de leite por dia e isto representa apenas $7,18 \%$ da produção. Por outro lado $2,37 \%$ dos produtores produzem mais do que 500 litros por dia, o que representa $25,53 \%$ da produção. Esta dualidade pode ser observada na maioria dos estados brasileiros produtores de leite.

A partir do número de produtores do mercado inspecionado, bem como os estratos de localização e tamanho da produção das propriedades, determinou-se a amostra de 485 produtores. A seguir a amostra foi dividida em dois grupos: um de 194 produtores, aos quais foram aplicados questionários quantitativos, cujo objetivo principal era a análise financeira das empresas rurais entrevistadas, e outro de 291 produto- 
res, aos quais foram aplicados questionários qualitativos, cujo objetivo principal era traçar o perfil do empresário e da sua empresa.

Após determinar o tamanho da amostra, esta foi dividida entre os 17 principais municípios produtores de leite do estado. Por exemplo, 11,41\% dos entrevistados tinham propriedades no município de Valença.

Os dados foram coletados de janeiro a dezembro de 2002. Após o preenchimento dos questionários os dados foram processados, formando duas grandes matrizes, sendo uma com dados quantitativos e outra com dados qualitativos.

\section{Resultados e Discussão}

\subsection{Parâmetros estimados}

O modelo de custo translogarítmico é um instrumento bastante utilizado na análise das economias de escala. A diferenciação da função custo resulta na estimação direta da elasticidade de custos em relação à produção (EC*). A partir deste resultado, obtêm-se os índices de economias de escala (IES) subtraindo-se EC* da unidade.

Considera-se neste estudo, além do modelo de custos translog (modelo A) mais quatro modelos: $\mathrm{O}$ modelo $\mathrm{B}$ impõe homoteticidade à função de produção associada; o modelo C, homogeneidade; o modelo D impõe elasticidade de substituição unitária e o modelo E, homogeneidade e elasticidade de substituição unitária (o que resulta na função custo Cobb-Douglas). As fórmulas para os índices de economias de escala (IES), para cada modelo são apresentadas na Tabela 1.

Tabela 1. Índice de economias de escala (IES) para os modelos de A a E

\begin{tabular}{ll}
\hline IES (A) & $1-\left(\beta_{y}+\beta_{y y} \ln Y+\beta_{k y} \ln w_{k}+\beta_{t y} \ln w_{t}+\beta_{m y} \ln w_{m}+\beta_{d y} \ln w_{d}\right)$ \\
IES (B) & $1-\left(\beta_{y}+\beta_{y y}\right)$ \\
IES (C) & $1-\beta_{y}$ \\
IES (D) & $1-\left(\beta_{y}+\beta_{y y} \ln Y+\beta_{k y} \ln w_{k}+\beta_{t y} \ln w_{t}+\beta_{m y} \ln w_{m}+\beta_{d y} \ln w_{d}\right)$ \\
IES (E) & $1-\beta_{y}$
\end{tabular}

Fonte: Chr` ensen e Greene (1976) 
A Tabela 2 mostra os parâmetros que foram obtidos, por máxima verossimilhança, para os cinco modelos considerados ${ }^{2}$. O modelo completo, sem restrições, translog A, resultou na estimativa direta de 15 coeficientes, sendo 13 deles estatisticamente diferentes de zero ao nível de significância de $1 \%$ e 2 ao nível de $20 \%$. Por meio da imposição das restrições de simetria e homogeneidade, foram calculados os seis parâmetros restantes do modelo sendo todos significativos a $1 \%$.

As estatísticas $t$ para os parâmetros de não homoteticidade $\left(\beta_{\mathrm{IY}}\right)$ e elasticidade de substituição unitária $\left(\beta_{\mathrm{IJ}}\right)$ para o modelo A (translog) sugerem que nem a hipótese de homoteticidade nem a elasticidade de substituição unitária são consistentes com o conjunto de dados. Esta suposição é confirmada pelo teste de Wald, que tem seus resultados apresentados na Tabela 3. As estatísticas do teste de $\chi^{2}$ para os modelos B, C, D e E sugerem a rejeição estatística das hipóteses realizadas com respeito à função de produção de: homoteticidade, homogeneidade, elasticidade de substituição unitária e homogeneidade com elasticidade de substituição unitária.

Portanto, o modelo de custos translog (modelo A) - que permite não homoteticidade, não homogeneidade e elasticidades de substituição não unitária à estrutura de produção associada - é o mais adequado para representar a estrutura de custos de produção de leite nos estados analisados.

$\mathrm{O}$ valor do parâmetro $\beta_{\mathrm{Y}}=-4,0985$, observado para o modelo $\mathrm{A}$ indica forte tendência inicial de economias de escala. Os valores observados para os modelos $\mathrm{C}$, $\mathrm{D}$ e $\mathrm{E}$ não foram significativos. O parâmetro observado no modelo B foi significativo em nível de $10 \%$ de probabilidade. $\mathrm{O}$ valor observado no parâmetro $\beta_{\mathrm{YY}}$ foi positivo no modelo A, o que confirma a hipótese de que as economias de escala diminuem à medida que aumenta a produção. Nos modelos B e D, estes valores não foram significativos, e o nos modelos $\mathrm{C}$ e $\mathrm{E}$ eles não aparecem devido às restrições impostas a estes modelos.

${ }^{2} \mathrm{O}$ software utilizado para estimar o sistema de equações, proposto no presente trabalho, foi o EVIEWS - versão 3. As descrições dos comandos para estimação dos modelos e realização dos testes de hipóteses encontram-se em: SOARES, I. G., CASTELAR, I. Econometria Aplicada com o Uso do Eviews. 
Tabela 2. Coeficientes da função de custos para os modelos de A a E

\begin{tabular}{|c|c|c|c|c|c|c|}
\hline \multirow{2}{*}{$\begin{array}{l}\text { Parâ- } \\
\text { metros }\end{array}$} & \multirow{2}{*}{ Origem } & \multicolumn{5}{|c|}{ Modelo } \\
\hline & & A & B & $\mathrm{C}$ & $\mathrm{D}$ & $E$ \\
\hline$\beta_{0}$ & regressão & $21,7708^{*}$ & $1,3565^{*}$ & $1,8233^{*}$ & $-0,6303 \mathrm{NS}$ & $-0.0775 \mathrm{NS}$ \\
\hline$\beta_{\mathrm{K}}$ & regressão & $0,6744^{*}$ & $0,5260^{*}$ & $0,6133^{*}$ & $-0,0140 \mathrm{NS}$ & $-2.25 \mathrm{E}-07^{*}$ \\
\hline$\beta_{\mathrm{M}}$ & regressão & $0,3087^{*}$ & $0,3882^{*}$ & $0,1573^{*}$ & $1,0589 *$ & $-1.5594^{*}$ \\
\hline$\beta_{\mathrm{T}}$ & regressão & $0,1648^{*}$ & $0,3621^{*}$ & $0,1252^{*}$ & $1,3846^{*}$ & $1.2790^{*}$ \\
\hline$\beta_{\mathrm{C}}$ & $1-\beta_{\mathrm{K}}-\beta_{\mathrm{M}}-\beta_{\mathrm{T}}$ & $-0,1480^{*}$ & $-0,2764^{*}$ & $0,1041^{*}$ & $-1,4295^{*}$ & $1.2804^{*}$ \\
\hline$\beta_{\mathrm{Y}}$ & regressão & $-4,0985^{*}$ & $1,32 \mathrm{E}-06^{* *}$ & 2,75E-08 NS & $9,10 \mathrm{E}-06 \mathrm{NS}$ & $0.6243 \mathrm{NS}$ \\
\hline$\beta_{\mathrm{KY}}$ & regressão & $-0,0063 * * *$ & $\cdots$ & $\cdots$ & $3,92 \mathrm{E}-06^{*}$ & $\cdots$ \\
\hline$\beta_{\mathrm{MY}}$ & regressão & $-0,0151^{*}$ & $\cdots$ & $\ldots$ & $-6,19 \mathrm{E}-06^{*}$ & $\ldots$ \\
\hline$\beta_{\mathrm{TY}}$ & regressão & $-0,0046^{* * *}$ & $\cdots$ & $\cdots$ & $-8,82 \mathrm{E}-06^{*}$ & $\cdots$ \\
\hline$\beta_{\mathrm{CY}}$ & $-\beta_{\mathrm{KY}}-\beta_{\mathrm{MY}}-\beta_{\mathrm{TY}}$ & $0,0261 *$ & $\cdots$ & $\cdots$ & $0.000011^{*}$ & $\cdots$ \\
\hline$\beta_{\mathrm{YY}}$ & regressão & $0,4179 *$ & $-3,66 \mathrm{E}-12 \mathrm{NS}$ & $\cdots$ & $4,22 \mathrm{E}-12 \mathrm{NS}$ & $\cdots$ \\
\hline$\beta_{\mathrm{KK}}$ & regressão & $0,1327^{*}$ & $0,0577^{* *}$ & $0,0844^{*}$ & $\cdots$ & $\cdots$ \\
\hline$\beta_{\mathrm{MM}}$ & regressão & $-0,0621^{*}$ & $-0,4997^{*}$ & $-0,0483^{*}$ & $\cdots$ & $\cdots$ \\
\hline$\beta_{\mathrm{TT}}$ & regressão & $0,0446^{*}$ & $0,0885^{*}$ & $0,0511^{*}$ & $\cdots$ & $\cdots$ \\
\hline$\beta_{\mathrm{CC}}$ & $-\beta_{\mathrm{KC}}-\beta_{\mathrm{MC}}-\beta_{\mathrm{TC}}$ & $-0.1211^{*}$ & $-1,6583^{*}$ & $0,139^{*}$ & $\cdots$ & $\cdots$ \\
\hline$\beta_{\text {Км }}$ & regressão & $-0,0946^{*}$ & $-0,6922^{*}$ & $-0,0720^{*}$ & $\cdots$ & $\cdots$ \\
\hline$\beta_{\mathrm{KT}}$ & regressão & $-0,0828^{*}$ & $0,0520^{*}$ & $0,0585^{*}$ & $\cdots$ & $\cdots$ \\
\hline$\beta_{\mathrm{KC}}$ & $-\beta_{\mathrm{KK}}-\beta_{\mathrm{KM}}-\beta_{\mathrm{KT}}$ & $0,0447^{*}$ & $0,5824^{*}$ & $-0,0709 *$ & $\cdots$ & $\cdots$ \\
\hline$\beta_{\mathrm{MT}}$ & regressão & $0,0592 *$ & $-0,0122 \mathrm{NS}$ & $0,0394^{*}$ & $\cdots$ & $\cdots$ \\
\hline$\beta_{\mathrm{MC}}$ & $-\beta_{\mathrm{KM}}-\beta_{\mathrm{MM}}-\beta_{\mathrm{MT}}$ & $0,0974 *$ & $1,2042 *$ & $0,0809^{*}$ & $\cdots$ & $\cdots$ \\
\hline$\beta_{\mathrm{TC}}$ & $-\beta_{\mathrm{KT}}-\beta_{\mathrm{MT}}-\beta_{\mathrm{TT}}$ & $-0,0210^{*}$ & $-0,1283^{*}$ & $-0,1490^{*}$ & $\cdots$ & $\cdots$ \\
\hline $\begin{array}{l}\text { Restri- } \\
\text { ções }\end{array}$ & - & Nenhuma & (3) & (4) & (6) & (10) \\
\hline
\end{tabular}

Fonte: Dados da pesquisa

Nota: Significância da estatística $t$ (de student) ${ }^{* *}$ significativo a $20 \%$; ** significativo a $10 \%$, * significativo a $1 \%$; NS = Não significativo a $20 \%$ 
Tabela 3. Teste de hipóteses para imposição de homoteticidade, homogeneidade e elasticidade de substituição unitária a função de produção associada à equação de custo.

\begin{tabular}{lcccc}
\hline & \multicolumn{4}{c}{ Restrições impostas ao modelo A } \\
\cline { 2 - 5 } & Homoteticidade & Homogeneidade & $\begin{array}{c}\text { Elasticidade de } \\
\text { substituição } \\
\text { unitária }\end{array}$ & $\begin{array}{c}\text { Homogeneidade } \\
\text { e elasticidade de } \\
\text { substituição unitária }\end{array}$ \\
\hline $\begin{array}{l}\text { Restrições } \\
\text { impostas } \\
\begin{array}{l}\text { Total de } \\
\text { restrições }\end{array}\end{array}$ & $\beta_{\mathrm{IY}}=0$ & $\begin{array}{c}\beta_{\mathrm{IY}}=0 ; \\
\beta_{\mathrm{YY}}=0\end{array}$ & $\beta_{\mathrm{IJ}}=0$ & $\begin{array}{c}\beta_{\mathrm{IY}}=0 ; \beta_{\mathrm{YY}}=0 ; \\
\beta_{\mathrm{IJ}}=0\end{array}$ \\
$\begin{array}{l}\text { Valor calculado } \\
\text { do } \chi^{2}\end{array}$ & 3 & 4 & 6 & 10 \\
\hline
\end{tabular}

Fonte: Dados da pesquisa

Nota: *** significativo a $20 \%$; ** significativo a $10 \%,{ }^{*}$ significativo a $1 \%$; NS = Não significativo a $20 \%$

Considerando o modelo A como sendo o mais indicado para os propósitos do estudo, verificou-se as condições para atender às propriedades da função custo, de homogeneidade linear nos preços, monotonicidade e convexidade nos preços dos fatores. Nota-se que todas as condições são atendidas pela função de custo translogarítmica estimada (modelo A). A monotonicidade da função é comprovada pelo comportamento das parcelas de custos, que se apresentam todas positivas. Estas parcelas foram calculadas pela média aritmética da amostra, sendo que os valores encontrados foram:

Parcela de custos com capital: $\mathrm{SK}=0,62$;

Parcela de custos com terra: $\mathrm{ST}=0,13$;

Parcela de custos com máquinas: $\mathrm{SM}=0,15$;

Parcela de custos com dispêndio: $\mathrm{SD}=0,10$.

\section{2 Índice de economias de escala para a amostra de produtores}

Estimativas de economias de escala (IES) podem ser calculadas para cada produtor individualmente, em nível observado de produção e preços de fatores, por meio das fórmulas apresentadas na Tabela 4. Adota-se, no entanto, o procedimento descrito em Christensen e Greene (1976) e divide-se a amostra dos produtores em grupos, de acordo com 
o nível de produção. Optou-se por dividir a amostra em sete grupos. Os IES são calculados para a média de produção e preços de cada grupo como segue: 1) produção média de 6,36 mil litros de leite por ano; 2) 10,05 mil litros/ano; 3) 14,35 mil litros/ano; 4) 21,32 mil litros/ano; 5) 37,50 mil litros/ano; 6) 64,03 mil litros/ano; 7) 186,99 mil litros/ano. A Tabela 4 mostra os resultados dos IES para os grupos. Optou-se por apresentar apenas os resultados do modelo translogarítmico (modelo A) devido à maior consistência das economias de escala observadas neste modelo.

Tabela 4. Índice de economias de escala (IES) para diferentes níveis de produção de leite na amostra analisada para a função de custos translog

\begin{tabular}{cccccccc}
\hline \multicolumn{7}{c}{ Nível de produção (em 1.000 litros de leite por ano) } \\
\hline & 6,36 & 10,05 & 14,35 & 21,32 & 37,50 & 64,03 & 186,99 \\
Modelo A & 1,37 & 1,18 & 1,03 & 0,87 & 0,63 & 0,41 & $-0,04$ \\
\hline
\end{tabular}

Fonte: Dados da pesquisa

Conforme esperado, as economias de escala diminuem com o aumento da produção. No entanto, nota-se que essa redução ocorre de forma bem suave. Os produtores com média de 186 mil litros por ano apresentam deseconomias de escala, mas se encontram próximos do ponto de custo médio mínimo, que é o ponto onde os produtores deixam de realizar economias de escala para realizar deseconomias de escala. Este ponto situa-se próximo do nível de produção de 178 mil litros. Portanto, estão bem próximos do ponto de ótimo.

\subsection{Curva de custo médio para a amostra de produtores de leite}

Os parâmetros do modelo estimado permitem a construção da curva de custo médio da amostra (Figura 1). A curva de custo médio é derivada avaliando-se sua função para os diferentes níveis de produção observados, mantendo-se os preços dos fatores fixos na média da amostra. Observa-se que a curva de custo médio correspondente ao modelo translog apresenta a forma clássica de $\mathrm{U}$, com os custos decrescendo com o nível de produção até o ponto de custo mínimo da curva de custo médio. Além deste ponto tem-se deseconomias de escala e o produtor passa a operar no ramo 
crescente da curva de custo médio, que é o ramo que permite ao produtor maximizar seu lucro. Nota-se na Figura 1 que os produtores que estão trabalhando no ponto de custo mínimo apresentam o nível de produção em torno de 178 mil litros de leite por ano, o que gera uma média diária de 487 litros por dia. Este resultado revela que a maioria dos produtores da amostra, nos três estados, não está maximizando sua renda, ou seja, pode aumentar a produção e caminhar para próximo do ponto de custo mínimo. Portanto, a faixa de economias de escala vai até o nível de 487 litros/dia. Apenas 3,5\% do total de produtores apresentaram produção média superior a este valor. Vale lembrar que a média dos estabelecimentos em cada estado não ultrapassou 100 litros por dia.

Do outro lado tem-se a grande massa de $96,5 \%$ da amostra que situam-se na faixa de produção que apresenta economias de escala. No entanto, este ramo que apresenta retornos crescentes à escala, pode ser subdividido ainda em duas partes. Uma parte composta por produtores que estão próximos do ponto de custo médio mínimo e tem sua produção entre 70 e 180 mil litros por ano, o que corresponde de 250 a 500 litros de leite por dia. Este grupo representa apenas 10,6\% do total da amostra. O outro grupo, que representa $85,9 \%$ do total da amostra, apresentou elevados níveis de economias de escala e a produção média diária foi inferior a 184 litros. Este grupo pode reduzir de forma significativa seu custo médio total com incrementos na produção. Sobre este grupo incidem severamente custos como depreciação e remuneração do capital imobilizado na atividade.

A caracterização dos tipos de retornos ajuda a compreender o problema desses produtores. Se o retorno for crescente à escala, como é o caso de grande parte da amostra, a produção é menor do que a quantidade que maximiza o lucro, ou seja, a produção pode expandir até o ramo dos retornos decrescentes. As causas deste fenômeno podem ser restrições externas, por exemplo, o crédito, como responsável pelo fato da produção estar menor no ponto de ótimo. As restrições podem também estar relacionadas com capital humano do produtor. E ainda existem outras restrições importantes que serão discutidas a seguir:

- A indivisibilidade de insumos é uma restrição que merece destaque. O modelo de competição perfeita apresenta algumas pressuposições implícitas e a mais restritiva delas é que cada insumo é divisível. 
Neste caso considera-se que é possível alugar terras, equipamentos, contratar horas de trabalho, de acordo com o plano ótimo de produção. No entanto, as imperfeições do mercado levam à apropriação da terra em níveis exagerados, conseqüentemente, o tamanho da exploração fica também condicionada ao uso das máquinas e equipamentos que nem sempre são disponibilizados aos produtores.

- Mercado imperfeito de produtos e insumos é um componente que impede o crescimento dos pequenos produtores. O mercado de produto e insumos favorece os grandes produtores. Em geral, o preço dos produtos e insumos está relacionado aos volumes de compra e venda, onde os pequenos pagam mais pelos insumos e recebem menos pelos produtos. Esta dinâmica pode fazer com que os pequenos fiquem com a produção estagnada e deixem seus estabelecimentos no longo prazo.

Figura 1. Curva de custo médio para os produtores de leite nos três estados analisados - modelo Translog

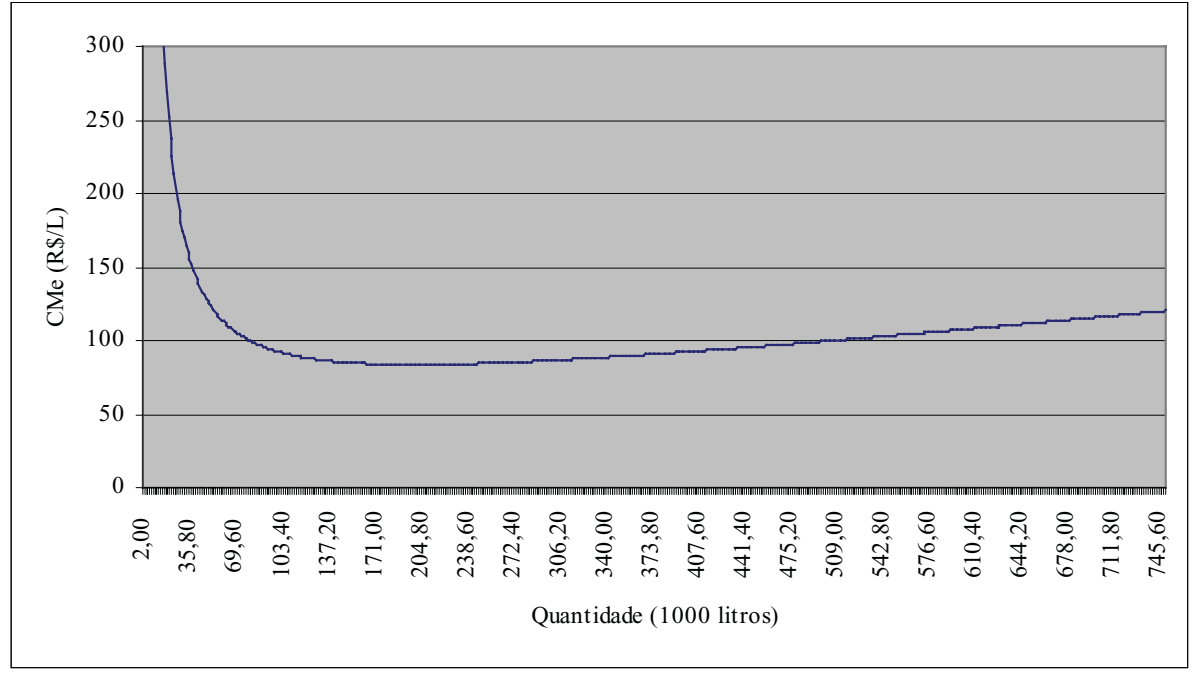

Vale lembrar que, se não houver obstáculos que impeçam aos produtores de caminharem para a escala ótima de produção, eles devem convergir para índices de eficiência iguais.

$\mathrm{O}$ acesso à assistência técnica de qualidade também depende de recursos para pagar os serviços. Quem produz maior volume tem maior 
facilidade de obter os recursos necessários, razão pela qual ele adota tecnologias mais eficientes.

O crédito é um fator de grande importância para a rentabilidade do negócio. Em geral, a maior produção sofre menos restrições bancárias, assim o racionamento externo do crédito é menos severo e financiado a taxa de juros menores com prazos mais dilatados. Por tudo isso a maior produção tem mais facilidade para evoluir para a escala ótima.

\section{Conclusões}

Nos últimos 15 anos, as regiões Centro-Oeste e Norte vêm ganhando mais espaço no agronegócio nacional, e o leite é um dos produtos que teve sua produção incrementada nestas regiões por apresentar vantagens comparativas em relação aos custos de produção. Se estes produtores conseguirem produzir leite com custos mais baixos do que as regiões tradicionalmente reconhecidas pela atividade, uma nova configuração para a pecuária de leite nacional pode estar sendo projetada, no longo prazo.

Os resultados das estimativas das economias de escala para o modelo de custo translog mostram uma escala ótima em torno de 490 litros por dia. No entanto, apenas 3,5\% dos produtores da amostra encontram-se neste nível de produção ou acima. Portanto, os produtores analisados podem aumentar a produção o que permitirá a redução do custo médio mínimo.

Em relação ao Estado do Rio de Janeiro nota-se uma maior utilização de tecnologias que favorecem o aumento da produção, e conseqüentemente, a redução do custo médio. A utilização de um modelo específico para cada região poderia diagnosticar a realização de economias de escala pelos produtores deste estado, tendo em vista que foi a região onde foram observados maiores níveis de produção.

Se por um lado regiões como Centro-Oeste e Norte apresentam vantagens em relação ao baixo custo de produção, os produtores do Rio de Janeiro mostram maior grau de especialização na atividade leiteira no que diz respeito ao uso de insumos e no grau de sangue dos animais, que são fatores que assumem grande importância como fonte de crescimento da produção de leite. 
Os resultados mostram que falta de conhecimento da atividade e a baixa capacidade de custeio dos produtores são fatores que contribuem para a baixa produção de leite dos estabelecimentos. Portanto, neste estado pode-se aumentar a produção com a realização de políticas que ofereçam assistência técnica e facilitem o acesso ao crédito para produtores de leite.

Deve se destacar que os resultados obtidos para as economias de escala são influenciados neste sentido, ou seja, o Estado do Rio de Janeiro apresenta diferentes características produtivas e tecnológicas em relação aos outros estados analisados (Rondônia e Tocantins).

Apesar de o modelo não explicar o porquê dos produtores não conseguirem realizar produções mais elevadas, alguns fatores podem ser apontados como barreiras ao crescimento da produção:

a) O baixo nível de dispêndios diretos em relação ao montante de capital imobilizado na atividade. O nível de custeio é que torna o capital fixo produtivo. Se ele é baixo, aumenta ociosidade do capital imobilizado na atividade.

b) As imperfeições do mercado de crédito e insumos, onde os estabelecimentos com níveis de produção mais elevados têm facilidades para comprar, taxas de juros reduzidas e maiores prazos para fazerem seus pagamentos, enquanto que os pequenos não podem desfrutar destes incentivos.

c) Muitos produtores tomam a decisão de utilizar no rebanho animais com características da raça zebu, ao invés do holandês que possui maior aptidão para a produção de leite. Esta estratégia se dá pelo fato dos animais da raça zebu apresentarem maior resistência a condições adversas e facilidade no manejo, apresentando, no entanto, níveis de produção menos expressivos que o gado holandês.

d) O baixo conhecimento para o uso de novas tecnologias também favorece a estagnação da produção. De forma que se o produtor não conhece medidas de manejo e tecnologias que permitam aumentar a produção, ele estará sempre receoso quanto à inovação tecnológica no seu sistema de produção.

e) A pulverização da produção é outro fator que contribui para que os produtores, principalmente os pequenos, fiquem na mesma situação e não apresentem aumentos na produção. A organização 
em cooperativas poderia permitir, aos produtores, maior poder de barganha na compra de insumos e na venda do leite para o mercado, garantindo melhores preços.

Percebe-se, assim, como é difícil a situação daqueles produtores que estão com o nível de produção muito abaixo do que leva ao custo médio mínimo, principalmente no que diz respeito ao longo prazo. Nesta situação, os estabelecimentos podem até estar pagando suas despesas com custeio, mas a não remuneração do capital condena estes estabelecimentos a fecharem suas portas no longo prazo.

A preocupação que surge diante deste quadro é que pode acontecer com estas famílias, que, geralmente possuem baixa escolaridade e, portanto têm poucas chances de conseguirem um trabalho digno nas cidades.

\section{Referências bibliográficas}

ALBUQUERQUE, M. C. C. de. Uma análise translog sobre mudança tecnológica e efeitos de escala: um caso de modernização eficiente. Pesquisa e Planejamento Econômico, v. 1, n. 17, p. 191-220, 1987.

ALVES, E. Retornos à escala e mercado competitivo: teoria e evidências empíricas. Revista de Economia e Agronegócio, v. 2, n. 3, p. 311-334, 2004.

ALVES, E.; SOUZA, G. da S.; BRANDÃO, A. S. P. A situação do produtor que tem menos de cem hectares. Revista de Política Agrícola, ano X, n. 1, p. 27-36, 2001.

BINSWANGER, H. P. A cost function approach to the measurement of elasticities of factor demand and elasticities of substitution. American Journal of Agricultural Economics, v. 56, p. 377-386, 1974.

BUARQUE, C. Avaliação econômica de projetos. Rio de Janeiro: Campus, 1991. 266p.

CHAMBERS, R. G. Applied production analysis: a dual approach. 4. ed. New York: Cambridge University Press. 1994. 331 p.

CHIANG, A. C. Matemática para economistas. Tradução: Roberto Campos Moraes. São Paulo: Makron Books, Pearson Education. 2004. 684 p. 
CHRISTENSEN, L. R.; GREENE, W. H. Economies of Scale in US electric power generation. Journal of Political Economy, v. 84, n. 4, p. 655676, 1976.

DENZIL, B. R.; GOMES, A. P. Eficiência da pequena produção de leite no Estado de Rondônia In: Congresso Brasileiro de Economia e Sociologia Rural, 2004, Cuiabá. Anais... Cuiabá: UFMT, 2004.

FARIA, V. P. Produção e cooperativas de produtores de leite no Brasil. In: Seminário: As cooperativas e a produção de leite no ano 2000. Belo Horizonte, 1995. Anais. Belo Horizonte: OCEMG, 1995. p. 29-40.

FERREIRA, A. H. Eficiência de sistemas de produção de leite: uma aplicação da análise envoltória de dados na tomada de decisão. 2002. 120 p. Dissertação (Mestrado) - Universidade Federal de Viçosa, Viçosa - MG.

GARCIA, L. A. F. Economias de escala na produção de frangos de corte no Brasil. 2003. 136 p. Tese (Doutorado) - Escola Superior de Agricultura "Luiz de Queiroz", Universidade de São Paulo, Piracicaba -SP.

GOMES, A. L. Determinantes da queda do preço do leite recebido pelo produtor: uma abordagem de curto e longo prazo. $2002.47 \mathrm{p}$. Dissertação (Mestrado) - Universidade Federal de Viçosa, Viçosa - MG.

GOMES, A. P., BAPTISTA, A. J. M. S., WENDLING, L. L. Fatores discriminantes do desempenho regional da produção de leite. In: Congresso Brasileiro de Economia e Sociologia Rural, 43, 2005, Ribeirão Preto. Anais... Ribeirão Preto, USP, 2005.

GOMES, S. T. Evolução e perspectivas da produção de leite no Brasil. In: GOMES A. T.; LEITE, J. L. B.; CARNEIRO A.V. (Ed). O Agronegócio do Leite no Brasil. Juiz de Fora: Embrapa Gado de Leite, 2001. p. 49-61

GREENE, W. T. Econometric analysis. 4. ed, New Jersey: Prentice Hall, 2000, 1004 p.

INSTITUTO BRASILEIRO DE GEOGRAFIA E ESTATISTISCA (IBGE). Censo Agropecuário 1995/96 www.ibge.gov.br (1 fev de 2005)

INSTITUTO BRASILEIRO DE GEOGRAFIA E ESTATISTISCA (IBGE). Pesquisa da pecuária municipal www.ibge.gov.br (10 mar de 2005) 
MARTINS, P. C. Políticas públicas e mercados deprimem o resultado do sistema agroindustrial do leite. 2002. 217 p. Tese (Doutorado) Escola Superior de Agricultura "Luiz de Queiroz”, Universidade de São Paulo, Piracicaba - SP.

PEREIRA FILHO, C. A. Eficiência econômica da pequena produção familiar no Recôncavo do Estado da Bahia: uma análise não paramétrica de fronteiras de produção multi-produto. 2000. 122 p. Tese (Doutorado) - Escola Superior de Agricultura "Luiz de Queiroz", Universidade de São Paulo, Piracicaba - SP.

RAY, S. C. A translog cost function analysis of US. Agriculture, 193977. American Journal of Agricultural Economics, v. 56, n. 1, p. 490-498, 1982.

ROBERTS, D. B.; GOMES A. P. Eficiência da pequena produção de leite no Estado de Rondônia In: Congresso Brasileiro de Economia e Sociologia Rural, 42, 2004, Cuiabá. Anais... Cuiabá, UFMT, 2004.

RODRIGUES, G. V. Estruturação e desafios do setor de produção de leite. In: CASTRO, M. C. .D.; MARTINS, P. C. (Ed.) Organização da produção primária: um desafio para a indústria de laticínios. Juiz de Fora: Epamig/ILCT, 1999. p. 36-40.

SOUZA, D. P. H. de Avaliação de Métodos Paramétricos e não paramétricos na análise da eficiência da produção de leite. 2003. 147 p. Tese (Doutorado) - Escola Superior de Agricultura "Luiz de Queiroz", Universidade de São Paulo, Piracicaba - SP.

Recebido em agosto de 2006 e revisto em abril de 2007. 